\title{
IMAGE SEGMENTATION WITH A FINITE ELEMENT METHOD
}

\author{
Blaise Bourdin ${ }^{1}$
}

\begin{abstract}
The Mumford-Shah functional for image segmentation is an original approach of the image segmentation problem, based on a minimal energy criterion. Its minimization can be seen as a free discontinuity problem and is based on $\Gamma$-convergence and bounded variation functions theories. Some new regularization results, make possible to imagine a finite element resolution method. In a first time, the Mumford-Shah functional is introduced and some existing results are quoted. Then, a discrete formulation for the Mumford-Shah problem is proposed and its $\Gamma$-convergence is proved. Finally, some numerical results, computed from both artificial and real images are presented and discussed.

Résumé. La fonctionelle de Mumford-Shah est une approche originale du problème de la segmentation d'images, basée sur un critère d'énérgie minimale. Sa minimisation peut être vue comme un problème de discontinuités libres et repose alors sur les théories de la $\Gamma$-convergence et des fonctions à variations bornées. Des résultats de régularisation récents ont permi d'envisager une méthode de résolution, à base d'éléments finis. Dans un premier temps, le modèle est présenté et des résultats existants sont cités. Ensuite, une formulation discrète du problème de Mumford-Shah est proposée et sa $\Gamma$-convergence prouvée. Enfin, des résultats numériques, issus de calculs sur des images synthétiques et réelles sont proposés et discutés.
\end{abstract}

AMS Subject Classification. 68U10, 65N30.

Received: November 4, 1997. Revised: February 18, 1998.

\section{INTRODUCTION}

Our aim is to minimize the functional proposed by Mumford and Shah [12] in the problem of image segmentation, namely

$$
\mathcal{E}(u, \Gamma)=\beta \int_{\Omega \backslash \Gamma}(u-g)^{2} d x+\int_{\Omega \backslash \Gamma}|\nabla u|^{2} d x+\alpha \mathcal{H}^{n-1}(\Gamma \cap \Omega)
$$

where $\Omega \subset \mathbb{R}^{2}$, an open bounded set, is the image domain, $g$ is the observed grey level of the image $(g \in$ $\left.L^{\infty}(\Omega ;[0,1])\right)$ and $u$ is a smooth approximation of $g\left(u \in \mathcal{C}^{1}(\Omega \backslash \Gamma)\right), \Gamma$ denotes the set of possible edges (a closed subset of $\Omega$ ) and $\mathcal{H}^{n-1}$ denotes the $n-1$-dimensional Hausdorff measure and $\alpha$ and $\beta$ are positive fixed parameters.

\footnotetext{
1 LPMTM, Institut Galilée, Université Paris Nord, avenue J.B. Clément, 93430 Villetaneuse, France. Present address: Department of Mathematics, Technical University of Denmark, 2800 Lyngby, Denmark. e-mail: bourdin@mat.dtu.dk
} 
In a formal way, $\mathcal{E}$ can be represented by an energy, associated to a function $u$, which must be smooth and close to the original image, except on a curve set $\Gamma$, called the edge set of the image.

This problem has been studied by several authors. First, De Giorgi, et al. [8] proposed an equivalent functional, $E$, with $u$ in $S B V(\Omega)$ and $\Gamma$ represented by $S_{u}$, the discontinuity set of $u$.

Then, Ambrosio and Tortorelli devised an approximation $E_{c}$ of $E$ by means of an elliptic functional that uses an additional variable $v$, representing (in some sense) $\mathcal{H}^{n-1}\left(S_{u}\right)$ and a small parameter, $c$, the relaxation parameter, via $\Gamma$-convergence. In view of the properties of $\Gamma$-convergence, the minimization of $E$ reduces to that of $E_{c}$, for small $c$. See $[1,2]$.

This work led to at least two different numerical approaches: Belletini and Coscia [3] proposed a discrete approximation $E_{c, h}^{B}$ of $E_{c}$ by piecewise linear functions, which lends itself to a finite element analysis of the problem, while Richardson and Mitter [13] implemented a gradient method.

In this study, we start from the functional $E_{c}$, propose a discrete approximation $E_{c, h}$ different from that proposed by Belletini and Coscia and implement a finite element method for the minimization of $E_{c, h}$.

The content of this paper is as follows:

Section 1 is entirely devoted to notation. In Section 2, we recall various results borrowed from the already quoted references. In Section 3, we present our discrete functional $E_{c, h}$ and prove its $\Gamma$-convergence to $E$ and the convergence of the minimum values and of the minimizers. In Section 4, we detail the numerical implementation of the computation. In Section 5, we present and discuss a few numerical results computed from both real and artificial images.

\section{Notation}

In $\mathbb{R}^{n},|\bullet|$ denotes the usual Euclidean norm, $d x$ or $\mathcal{L}^{n}$, the Lebesgue measure, and $\mathcal{H}^{k}$, the $k$-dimensional Hausdorff measure.

Set $\mathcal{E}(u, \Gamma)=\beta \int_{\Omega \backslash \Gamma}(u-g)^{2} d x+\int_{\Omega \backslash \Gamma}|\nabla u|^{2} d x+\alpha \mathcal{H}^{n-1}(\Omega \cap \Gamma)$ (the Mumford-Shah functional).

Let $B V(\Omega)$ be the space of functions $u \in L^{1}(\Omega)$ such that the distributional derivative of $\mathrm{u}$ can be represented by a regular Borel measure with finite total variation $D u: B(\Omega) \rightarrow \mathbb{R}^{n}$, where $B(\Omega)$ denotes the set of all Borel subsets of $\Omega$.

Let $S_{u}$ be the complement of the Lebesgue set of u, i.e.,

$$
S_{u}=\left\{x \in \Omega: \nexists z \in \mathbb{R} \text { such that } \lim _{\rho \rightarrow 0^{+}} \rho^{-n} \int_{B_{\rho}(x)}|u(x)-z| d x=0\right\}
$$

Denote by $S B V(\Omega)$, the space of functions $u$ in $B V(\Omega)$ such that $D u$ is absolutely continuous with respect to $\mathcal{L}^{n}+\mathcal{H}^{n-1}\left\llcorner S_{u}\right.$.

For further properties of $B V$ and $S B V$, see [10] and [1].

Denote by $\mathcal{R}$, the class of all piecewise $\mathcal{C}^{2}$ submanifolds of $\mathbb{R}^{n}$ of dimension $n-1$.

Denote by $\left\{S_{h}\right\}_{h}$, a regular family of partition of $\Omega$ into simplices.

Let $\mathbb{P}_{n}(\Omega)$ be the space of polynomials on $\Omega$ of total degree less or equal than $n$.

Denote by $V_{h}(\Omega) \subset H^{1}(\Omega)$, the linear finite element space over $S_{h}: v_{h} \in V_{h}$ if and only if $v_{h} \in \mathbb{P}^{1}(T), \forall T \in S_{h}$ and $v_{h} \in \mathcal{C}(\Omega)$.

Let $V_{h}(\Omega,[0,1])=\left\{v \in V_{h}(\Omega) ; \forall x \in \Omega, v(x) \in[0,1]\right\}$.

Let $\pi_{h}$ be the Lagrange projection operator over $V_{h}$, i.e. given $v \in \mathcal{C}(\Omega), \pi_{h}(v)$ is such that $\pi_{h}(v) \in V_{h}(\Omega)$ and for all nodes $s \in S_{h},\left(\pi_{h}(v)\right)(s)=v(s)$. 


\section{VARIOUS RESULTS}

\subsection{Extension to $S B V$ and equivalent problem}

Set

$$
E(u)=\beta \int_{\Omega}(u-g)^{2} d x+\int_{\Omega}|\nabla u|^{2} d x+\alpha \mathcal{H}^{n-1}\left(S_{u} \cap \Omega\right) .
$$

It is proved in [8] that $\min \{E(u), u \in S B V(\Omega)\}$ and $\min \left\{\mathcal{E}(u, \Gamma) ; \Gamma\right.$ closed subset of $\Omega$ and $\left.u \in \mathcal{C}^{1}(\Omega \backslash \Gamma)\right\}$ are equivalent problems. Firstly, $\inf \{E(u), u \in S B V(\Omega)\}$ is smaller than $\inf \{\mathcal{E}(u, \Gamma) ; \Gamma$ closed subset of $\Omega$ and $\left.u \in \mathcal{C}^{1}(\Omega \backslash \Gamma)\right\}$, which is immediate because, if $u \in \mathcal{C}^{1}(\Omega \backslash \Gamma) \cap L^{\infty}(\Omega), \int_{\Omega \backslash \Gamma}|\nabla u|^{2} d x<\infty$ and $\mathcal{H}^{n-1}(\Omega \cap \Gamma)<$ $\infty$, then $u \in S B V(\Omega)$ (Lemma 2.3 of [8]). Then the existence of $\min \{E(u), u \in S B V(\Omega)\}$ is established in Lemma 5.1 of [8]. Finally, if $u$ is a minimizer of $E$, on $S B V(\Omega)$, then (Lemma 5.2 of [8]) $u \in \mathcal{C}^{1}\left(\Omega \backslash \overline{S_{u}}\right)$ and $\mathcal{H}^{n-1}\left(\left(\overline{S_{u}} \cap \Omega\right) \backslash S_{u}\right)=0$, and the result is achieved.

The next step is to focus on the term $\mathcal{H}^{n-1}\left(S_{u} \cap \Omega\right)$. To this effect, $E$ is approximated in the sense of $\Gamma$-convergence by means of an elliptic functional.

Many such functionals have been proposed [1-3,13].

\section{2. $\Gamma$-convergence results}

The main idea is to use an additional functional variable $v: \Omega \rightarrow[0,1]$, that will "represent" the jump set $S_{u}$ and to define the relaxed functional $E_{c}(u, v)$, with $u$ and $v$ in a convenient space.

One sets

$$
F_{c}(u, v)=\left\{\begin{array}{l}
E_{c}(u, v) \text { if }(u, v) \in \operatorname{Dom}\left(E_{c}\right) \\
\infty \text { otherwise }
\end{array}\right.
$$

and

$$
F(u, v)=\left\{\begin{array}{l}
E(u) \text { if } u \in \operatorname{Dom}(E) \text { and } v=1 \text { a.e. } \\
\infty \text { otherwise. }
\end{array}\right.
$$

If $F_{c} \Gamma$-converges to $F$ when $c \rightarrow 0$, with respect to the $L^{2}(\Omega) \times L^{2}(\Omega ;[0,1])$ strong topology, and if the sequence of minimizers $\left(u_{c}, v_{c}\right)$ for $E_{c}$ is compact in $\operatorname{Dom}(E)$, then a subsequence of $\left(u_{c}, v_{c}\right)$ (still indexed by $c$ ) is such that $u_{c} \rightarrow u$ in $L^{2}(\Omega)$ and $v_{c} \rightarrow v$ in $L^{2}(\Omega)$ and $(u, v)$ minimizes $F$, i.e., $u$ minimizes $E$.

Remark. It is not necessary to establish the $\Gamma$-convergence of $F_{c}$ to $F$ so as to construct approximate minimizers. Indeed it suffices to prove that if $(u, v)$ is a minimizer for $F$, there exists $\left(u_{c}, v_{c}\right)$ in $\operatorname{Dom}\left(E_{c}\right)$, such that $\left(u_{c}, v_{c}\right) \rightarrow(u, v)$ in $L^{2}(\Omega)$ and $F(u, v) \geq \limsup F_{c}\left(u_{c}, v_{c}\right)$, that the lower inequality, $\liminf F_{c}\left(\bar{u}_{c}, \bar{v}_{c}\right) \geq F(\bar{u}, \bar{v})$ if $\left(\bar{u}_{c}, \bar{v}_{c}\right) \rightarrow(\bar{u}, \bar{v})$ in $L^{2}(\Omega)$, holds and that sequences of approximate minimizers are compact in $L^{2}(\Omega)$.

The first approximating functional of this kind was proposed in [1]. It is

$$
E_{c}(u, v)=\int_{\Omega}\left\{\beta(u-g)^{2}+\left(|\nabla u|^{2}+|\nabla v|^{2}\right)\left(1-v^{2}\right)^{\frac{2}{c}}+\frac{\alpha^{2}}{4 c^{2}} v^{2}\right\} d x
$$

with $W^{1,2}(\Omega) \times\left\{v \in W^{1,2}(\Omega) ; 0 \leq v \leq 1\right.$ a.e. $\} \subset \operatorname{Dom}\left(E_{c}\right) \subset \mathcal{B}(\Omega) \times\{v \in \mathcal{B}(\Omega) ; 0 \leq v \leq 1$ a.e. $\}$, where $\mathcal{B}(\Omega)$ is the set of Borelian mappings on $\Omega$.

The $\Gamma$-convergence takes place in $L^{2}(\Omega) \times L^{2}(\Omega ;[0,1])$ strong topology, and the total variation of $(1-v)^{1+1 / c}$ weakly converges in the sense of measures to $2 \mathcal{H}^{n-1}\left\llcorner S_{u}(B)\right.$, as $c \rightarrow 0$, i.e., $\mu_{c}$ defined as $\mu_{c}(B)=\left(1+\frac{1}{c}\right) \int_{B} v_{c}\left(1-v_{c}\right)^{\frac{1}{c}}\left|\nabla v_{c}\right| d x$ vaguely converges to $\mathcal{H}^{n-1}\left\llcorner S_{u}\right.$, as $c \rightarrow 0$.

Note that because of the term $\left(1-v^{2}\right)^{\frac{2}{c}}$, this functional cannot be easily discretized, as $c \rightarrow 0$. 
Then, in [2] the $\Gamma$-convergence, with respect to the $L^{2}(\Omega) \times L^{2}(\Omega ;[0,1])$ strong topology, of the following functional is addressed:

$$
E_{c}(u, v)=\int_{\Omega}\left\{\beta(u-g)^{2}+\left(v^{2}+k_{c}\right)|\nabla u|^{2}+\alpha\left(c|\nabla v|^{2}+\frac{(1-v)^{2}}{4 c}\right)\right\} d x,
$$

with $k_{c} \ll c$ and $\alpha>0$.

In (3), $\operatorname{Dom}\left(E_{c}\right)=\left\{L^{\infty}(\Omega) \cap \mathcal{C}^{1}(\Omega)\right\} \times\left\{L^{\infty}(\Omega ;[0,1]) \cap \mathcal{C}^{1}(\Omega ;[0,1])\right\}$ and $v$ plays the role that $1-v$ played in (2). This expression gave rise to two different numerical methodologies.

In [3] the $\Gamma$-convergence of a piecewise linear functional close to (3) is investigated.

It is of the form

$$
\begin{aligned}
& E_{c, h}^{B}(u, v)=\int_{\Omega}\left\{\beta \pi_{h}\left(\left(u-g_{c}\right)^{2}\right)+\left(v+k_{c}\right)|\nabla u|^{2}\right\} d x+\frac{2 \alpha}{\pi} M_{c, h}(v) \\
& \text { with } M_{c, h}(v)=\int_{\Omega}\left(c|\nabla v|^{2}+\frac{1}{4 c} \pi_{h}\left(1-v^{2}\right)\right) d x
\end{aligned}
$$

where $(u, v) \in V_{h}(\Omega) \times V_{h}(\Omega ;[0,1]), g_{c} \in \mathcal{C}_{0}^{\infty}(\Omega)$ approximates $g$, in a manner such that $g_{c} \rightarrow g$ in $L^{2}(\Omega)$, $\left\|g_{c}\right\|_{L^{\infty}(\Omega)} \leq\|g\|_{L^{\infty}(\Omega)}$ and $\left\|\nabla g_{c}\right\|_{L^{\infty}(\Omega)} \leq \frac{C}{c}$, which is feasible by convolution with an approximation of the identity (cf. e.g. [5], Sect. IV.4). If $h=o\left(k_{c}\right)$ and $k_{c}=o(c)$ then $F_{c, h}$ (defined from $E_{c, h}$ as $F_{c}$ was defined from $\left.E_{c}\right) \Gamma$-converges to $F$, with respect to the $L^{2}(\Omega) \times L^{2}(\Omega ;[0,1])$ strong topology, as $c \rightarrow 0$.

The functional $E_{c, h}$ is a good candidate for a finite element analysis of the problem. A numerical implementation of (4) has been recently proposed by [11] for synthetics images.

Yet another change from (3) is the formulation proposed in [13], namely,

$$
E_{c}(u, v)=\int_{\Omega}\left\{\beta(u-g)^{2}+\Phi(v)|\nabla u|^{2}+\alpha\left(c \Psi(v)|\nabla v|^{2}+\frac{(1-v)^{2}}{4 c}\right)\right\} d x .
$$

In (5), $\Phi$ and $\Psi$ are $\mathcal{C}^{1}$ functions with "good" properties, in which case, $F_{c}$ is shown in [2] to $\Gamma$-converge to $F$, with respect to the $L^{2}(\Omega) \times L^{2}(\Omega ;[0,1])$ strong topology.

Using this expression of $E_{c}$, a gradient descent algorithm is implemented in [13]: given $\left(u^{0}, v^{0}\right)$ and $c_{u_{n}}, c_{v_{n}}$, 2 scalars, compute:

$$
\left\{\begin{array}{l}
u^{n+1}=u^{n}-c_{u_{n}} \partial_{u} E_{c} \\
v^{n+1}=v^{n}-c_{v_{n}} \partial_{v} E_{c}
\end{array}\right.
$$

and update the scalars $c_{u_{n}}, c_{v_{n}}$, using $u_{n}, v_{n}, \Phi$ and $\Psi$.

The authors also propose dynamic scaling, setting $c=c(n), \beta=\beta(n)$, then using $c(n)$ as a stopping criterion, because the edge width is correlated with the value $c(n)$.

In the present paper, we propose to build a piecewise linear discretization of (3) proposed by Ambrosio and Tortorelli. We revisit the $\Gamma$-convergence of the discretized problem with respect to the $L^{2}(\Omega) \times L^{2}(\Omega ;[0,1])$ strong topology, the compactness of the sequence of the minimizers, then implement a finite element method so as to solve the discretized problem.

\section{The Finite ElEMENT METhoD FOR IMAGE SEGMENTATION}

\section{1. $\Gamma$-convergence and piecewise linear approximation}

Let us consider the functional $E_{c}$, proposed in [2], namely,

$$
E_{c}(u, v)=\int_{\Omega}\left\{\beta(u-g)^{2}+\left(v^{2}+k_{c}\right)|\nabla u|^{2}+\frac{2 \alpha}{\pi}\left(c|\nabla v|^{2}+\frac{(1-v)^{2}}{4 c}\right)\right\} d x .
$$


Set

$$
F_{c}(u, v)=\left\{\begin{array}{l}
E_{c}(u, v) \text { if }(u, v) \in H^{1}(\Omega) \times H^{1}(\Omega ;[0,1]) \\
+\infty \text { otherwise }
\end{array}\right.
$$

and let $E_{c, h}$, be the following

$$
E_{c, h}(u, v)=\int_{\Omega}\left\{\beta\left(u-g_{c, h}\right)^{2}+\left(v^{2}+k_{c}\right)|\nabla u|^{2}\right\} d x+\frac{2 \alpha}{\pi} M_{c, h}(v)
$$

where

$$
\begin{aligned}
& M_{c, h}(v)=\int_{\Omega}\left\{c|\nabla v|^{2}+\frac{((1-v))^{2}}{4 c}\right\} d x \\
& \text { and } g_{c, h}=\pi_{h}\left(g_{c}\right), \text { with } g_{c} \text { as in }(4),
\end{aligned}
$$

and

$$
F_{c, h}(u, v)=\left\{\begin{array}{l}
E_{c, h}(u, v) \text { if }(u, v) \in V_{h}(\Omega) \times V_{h}(\Omega ;[0,1]) \\
+\infty \text { otherwise }
\end{array}\right.
$$

Then, the following approximation theorem, which is a mere adaptation of Theorem 1.1 in [3] holds:

Theorem. Let $h \ll k_{c} \ll c$; then the minimum of $\left(E_{c, h}\right)$ converges to the minimum of $F$ as $c \rightarrow 0$.

Furthermore, if $\left(u_{c, h}, v_{c, h}\right)$ is a minimizer for $F_{c, h}$ then, possibly passing to a subsequence, $\left(u_{c, h}, v_{c, h}\right)$ converges strongly in $L^{2}(\Omega) \times L^{2}(\Omega ;[0,1])$ to a minimizer of $F$.

The proof of this theorem divides into three steps:

Step 1. - the lower inequality.

Let $h=o(c),(u, v) \in L^{\infty}(\Omega) \times L^{\infty}(\Omega ;[0,1])$ and for every $c>0$ let $\left(u_{c, h}, v_{c, h}\right) \in V_{h}(\Omega) \times V_{h}(\Omega ;[0,1])$ be such that the sequence $\left\{\left(u_{c, h}, v_{c, h}\right)\right\}_{c}$ converges to $(u, s)$ in $L^{2}(\Omega) \times L^{2}(\Omega ;[0,1])$.

Then

$$
F(u, v) \leq \liminf _{c \rightarrow 0} F_{c, h}\left(u_{c, h}, v_{c, h}\right)
$$

Step 2. - the upper inequality.

Let $h=o\left(k_{c}\right),(u, v) \in S B V(\Omega) \cap L^{\infty}(\Omega) \times L^{\infty}(\Omega ;[0,1])$. Then for every $c>0$, there exists $\left(u_{c, h}, v_{c, h}\right) \in$ $V_{h}(\Omega) \times V_{h}(\Omega ;[0,1])$ such that the sequence $\left\{\left(u_{c, h}, v_{c, h}\right)\right\}_{c}$ converges to $(u, v)$ in $L^{2}(\Omega) \times L^{2}(\Omega ;[0,1])$ and

$$
F(u, v) \geq \limsup _{c \rightarrow 0} F_{c, h}\left(u_{c, h}, v_{c, h}\right)
$$

Step 3. - compactness of the minimizers for $F_{c, h}$ and convergence of the minimum values.

The following properties pertaining to $E_{c, h}^{B}$, were proved in [3] (see Theorem 6.1 and 6.2), and will apply to $E_{c, h}$, at the expense of minor changes in the proofs.

Let $h=o\left(k_{c}\right)$, and $\left(u_{c, h}, v_{c, h}\right) \in V_{h}(\Omega) \times V_{h}(\Omega ;[0,1])$ be a minimum point of $F_{c, h}$, then, a subsequence (still indexed by $c)\left\{\left(u_{c, h}, v_{c, h}\right)\right\}_{c}$ converges in $L^{2}(\Omega) \times L^{2}(\Omega ;[0,1])($ as $c \rightarrow 0)$ to $(u, 1)$ where $u \in S B V(\Omega) \times L^{\infty}(\Omega)$

Further, assume that there exists at least a minimizer $\bar{u}$ of $F$. Then the minimum values of $F_{c, h}$ converge to the minimum value of $F$ as $c \rightarrow 0$. Moreover, any family $\left\{\left(u_{c, h}, v_{c, h}\right)\right\}_{c}$ of absolute minimizers of $\left\{F_{c, h}\right\}_{c}$ is relatively compact in $L^{2}(\Omega) \times L^{2}(\Omega ;[0,1])$, and each of its limit points minimizes $F$. 
Proof of step 1. Assume that $\liminf F_{c, h}\left(u_{c, h}, v_{c, h}\right)<\infty$, otherwise the result is trivial. Then,

$$
\liminf _{c \rightarrow 0} E_{c, h}\left(u_{c, h}, v_{c, h}\right)=\liminf _{c \rightarrow 0} E_{c}\left(u_{c, h}, v_{c, h}\right)
$$

Indeed,

But

$$
E_{c, h}\left(u_{c, h}, v_{c, h}\right)=E_{c}\left(u_{c, h}, v_{c, h}\right)+\beta \int_{\Omega}\left\{\left(u_{c, h}-g_{c, h}\right)^{2}-\left(u_{c, h}-g\right)^{2}\right\} d x
$$

$$
\int_{\Omega}\left\{\left(u_{c, h}-g_{c, h}\right)^{2}-\left(u_{c, h}-g\right)^{2}\right\} d x \leq \int_{\Omega} g_{c, h}^{2}-g^{2} d x+2 \int_{\Omega} u_{c, h} \cdot\left(g-g_{c, h}\right) d x .
$$

Since $g_{c, h}=\pi_{h}\left(g_{c}\right), g_{c} \in \mathcal{C}_{0}^{\infty}(\Omega)$ and $h=o(c), g_{c, h} \rightarrow g$ in $L^{2}$ when $c \rightarrow 0$.

Hölder's inequality implies

$$
\int_{\Omega}\left|u_{c, h} \cdot\left(g-g_{c, h}\right)\right| d x \leq\left(\int_{\Omega}\left|u_{c, h}\right|^{2} d x\right)^{1 / 2} \cdot\left(\int_{\Omega}\left|g-g_{c, h}\right|^{2} d x\right)^{1 / 2}
$$

and by virtue of the convergence of $u_{c, h}$ to $u$ and $g_{c, h}$ to $g$ in $L^{2}$ when $c \rightarrow 0$,

$$
\int_{\Omega}\left|u_{c, h} \cdot\left(g-g_{c, h}\right)\right| d x \rightarrow 0
$$

Thus $\liminf _{c \rightarrow 0} E_{c, h}\left(u_{c, h}, v_{c, h}\right)=\liminf _{c \rightarrow 0} E_{c}\left(u_{c, h}, v_{c, h}\right)$ and since $V_{h}(\Omega) \subset H^{1}(\Omega)$, one can use the $\Gamma$-convergence of $F_{c}$ to $F$ and conclude that $F(u, v) \leq \liminf _{c \rightarrow 0} F_{c, h}\left(u_{c, h}, v_{c, h}\right)$ and that the lower inequality holds.

Proof of step 2. In a first time, we will prove the upper inequality with the additional hypothesis, $S_{u} \in \mathcal{R}$, which has been supposed in [3]. Then, we will explain how to prevent from the use of it.

Let $h=o\left(k_{c}\right),(u, v) \in L^{\infty}(\Omega) \cap S B V(\Omega) \times L^{\infty}(\Omega ;[0,1])$. If $F(u, v)$ is not finite, the result is trivial, then we can suppose that $v \equiv 1$ and $u \in S B V(\Omega) \cap H^{1}\left(\Omega \backslash \bar{S}_{u}\right)$.

Set $\left(u_{c, h}, v_{c, h}\right) \in V_{h}(\Omega) \times V_{h}(\Omega ;[0,1])$ such that $\left(u_{c, h}, v_{c, h}\right) \rightarrow(u, v)$ in $L^{2}$ when $c \rightarrow 0, E_{c, h}\left(u_{c, h}, v_{c, h}\right) \leq C$ for all $c$ and $u_{c, h} \in W^{2, \infty}(S) ; \forall S \in S_{h}$.

One has:

$$
\begin{aligned}
E_{c, h}^{B}\left(u_{c, h}, v_{c, h}\right)-E_{c, h}\left(u_{c, h}, v_{c, h}\right)= & \beta \int_{\Omega} \pi_{h}\left(\left(u_{c, h}-g_{c}\right)^{2}\right)-\left(u_{c, h}-g_{c, h}\right)^{2} d x \\
& +\sum_{S \in S_{h}} \int_{S}\left(v_{c, h}+k_{c}\right)\left|\nabla u_{c, h}\right|^{2}-\left(v_{c, h}^{2}+k_{c}\right)\left|\nabla u_{c, h}\right|^{2} d x \\
& +\frac{\alpha}{2 . \pi \cdot c} \int_{\Omega} \pi_{h}\left(1-v_{c, h}^{2}\right)-\left(1-v_{c, h}\right)^{2} d x .
\end{aligned}
$$

We now prove that $I_{c, h}=\beta \int_{\Omega}\left|\pi_{h}\left(u_{c, h}^{2}\right)-\left(u_{c, h}\right)^{2}\right| d x \rightarrow 0$ when $c \rightarrow 0$. Indeed, $I_{c, h} \leq \sum_{S \in S_{h}}|S| \cdot \| \pi_{h}\left(u_{c, h}^{2}\right)-$ $u_{c, h}^{2} \|_{L^{\infty}(\Omega)}$, and, using [7], Theorem 3.1.5, $I_{c, h} \leq C^{\prime} \cdot h^{2} \cdot \sum_{S \in S_{h}}|S| \cdot\left\|D^{2}\left(u_{c, h}^{2}\right)\right\|_{L^{\infty}(S)}$.

Since $u_{c, h}$ is piecewise linear, $\left\|D^{2}\left(u_{c, h}^{2}\right)\right\|_{L^{\infty}(S)}=2 .\left\|\nabla u_{c, h} \otimes \nabla u_{c, h}\right\|_{L^{\infty}(S)}=2 .\left|\nabla u_{c, h}\right|^{2}$, for all $S \in S_{h}$ and since $E_{c, h}\left(u_{c, h}, v_{c, h}\right) \leq C$ for all $c$, one has $\sum_{S \in S_{h}} \int_{S}\left|\nabla u_{c, h}\right|^{2} d x \leq \frac{C}{k_{c}}$ thus $I_{c, h} \rightarrow 0$ as $c \rightarrow 0$.

The same kind of argument is used to prove that $\beta \int_{\Omega}\left|\pi_{h}\left(g_{c}^{2}\right)-\left(g_{c, h}\right)^{2}\right| d x \rightarrow 0$ and $\beta \int_{\Omega} \mid \pi_{h}\left(u_{c, h} . g_{c}\right)-$ $\left(u_{c, h} \cdot g_{c, h}\right)^{2} \mid d x \rightarrow 0$ when $c \rightarrow 0$.

Since $v_{c, h} \in V_{h}(\Omega ;[0,1]), v_{c, h}^{2} \leq v_{c, h}$, and $\int_{\Omega}\left(v_{c, h}+k_{c}\right)\left|\nabla u_{c, h}\right|^{2}-\left(v_{c, h}^{2}+k_{c}\right)\left|\nabla u_{c, h}\right|^{2} d x \geq 0$. Further, since $\pi_{h}$ is a linear projection, $\pi_{h}\left(v_{c, h}^{2}\right) \leq \pi_{h}\left(v_{c, h}\right)=v_{c, h}, \pi_{h}\left(1-v_{c, h}^{2}\right) \geq 1-v_{c, h} \geq\left(1-v_{c, h}\right)^{2}$ and $\sum_{S \in S_{h}} \int_{S} \pi_{h}(1-$ $\left.v_{c, h}^{2}\right)-\left(1-v_{c, h}\right)^{2} d x \geq 0$. Thus $\limsup _{c \rightarrow 0} E_{c, h}^{B}\left(u_{c, h}, v_{c, h}\right) \geq \limsup _{c \rightarrow 0} E_{c, h}\left(u_{c, h}, v_{c, h}\right)$

We now consider the sequence $\left(u_{c, h}, v_{c, h}\right)$ built in [3] for the upper inequality, and suppose that $S_{u} \in \mathcal{R}$; it is such that $\left(u_{c, h}, v_{c, h}\right) \rightarrow(u, v)$ in $L^{2}, \limsup _{c \rightarrow 0} E_{c, h}^{B}\left(u_{c, h}, v_{c, h}\right) \leq \int_{\Omega}|\nabla u|^{2}+\beta|u-g|^{2} d x+\alpha \mathcal{H}^{n-1}\left(S_{u}\right)$ and 
verifies the above mentioned hypothesis. Thus,

$$
\limsup _{c \rightarrow 0} E_{c, h}\left(u_{c, h}, v_{c, h}\right) \leq \int_{\Omega}|\nabla u|^{2}+\beta|u-g|^{2} d x+\alpha \mathcal{H}^{n-1}\left(S_{u}\right) .
$$

We now explain how to prevent from the use of the hypothesis $S_{u} \in \mathcal{R}$ :

In [12] Mumford and Shah conjectured that the minimizing set $\Gamma$ of $\mathcal{E}$ should be a finite union of $\mathcal{C}^{1}$ hypersurfaces. Some recent works partially proved this conjecture:

In [4], Bonnet proved it in the 2-dimensional case with the following additional hypothesis: $\Gamma$ has a finite number of connected components.

Dibos and Séré proved in [9], Theorem 1.7, that if $(u, \Gamma)$ is a minimum point of $\mathcal{E}$ then $\forall \epsilon>0, \exists\left(u_{\epsilon}, \Gamma_{\epsilon}\right)$ where $\Gamma_{\epsilon}$ is a finite union of smooth hypersurfaces (included in hyperplanes, spheres or cylinders), such that $u_{\epsilon} \in \mathcal{C}^{1}\left(\Omega \backslash \Gamma_{\epsilon}\right),\left\|u_{\epsilon}-u\right\|_{L^{1}} \leq \epsilon$ and $\mathcal{E}\left(u_{\epsilon}, \Gamma_{\epsilon}\right) \leq \mathcal{E}(u, \Gamma)+\epsilon$. This result was generalized by Dal Maso in [6], Lemma G.2, to all functions of $S B V(\Omega)$. Then, each function of $S B V(\Omega)$ can be approximated by functions $u_{\epsilon}$ such that $u_{\epsilon} \rightarrow u$ in $L^{1}, E\left(u_{\epsilon}\right) \leq E(u)+\epsilon$ and $S_{u_{\epsilon}} \in \mathcal{R}$. We apply the upper inequality to $u_{\epsilon}$ and by a diagonal sequence argument, conclude: If $h=o\left(k_{c}\right)$ and $(u, v) \in L^{\infty}(\Omega) \cap S B V(\Omega) \times L^{\infty}(\Omega ;[0,1])$, then there exist a sequence $\left(u_{c, h}, v_{c, h}\right) \in V_{h}(\Omega) \times V_{h}(\Omega ;[0,1])$ such that

$$
F(u, v) \geq \limsup _{c \rightarrow 0} F_{c, h}\left(u_{c, h}, v_{c, h}\right) .
$$

The proof of the third step follows step by step that of Theorems 6.1 and 6.2 in [3].

\section{NUMERICAL IMPLEMENTATION}

\subsection{The constraint on $v$}

A rapid investigation of the constraint $v \in[0,1]$, permits to simplify the numerical minimization of $E_{c, h}$. Suppose that $(u, v) \in V_{h}(\Omega)^{2}$ are minimizers for $E_{c, h}$. Set $\tilde{v}=\pi_{h}(\min (1, v))$, which is allowed $\operatorname{since} \min (1, v) \in$ $\mathcal{C}(\Omega)$.

Then $\int_{\Omega}|\nabla \tilde{v}|^{2} d x \leq \int_{\Omega}|\nabla v|^{2} d x, \int_{\Omega}(1-\tilde{v})^{2} d x \leq \int_{\Omega}(1-v)^{2} d x$ and $\int_{\Omega}\left(\tilde{v}^{2}+k_{c}\right)|\nabla u| d x \leq \int_{\Omega}\left(v^{2}+k_{c}\right)|\nabla u| d x$.

If $|\{x \in \Omega ; v(x)>1\}|>0$, then the two last inequalities are strict and $E_{c, h}(u, \tilde{v})<E_{c, h}(u, v)$, which is in contradiction with the hypothesis that $(u, v)$ are minimizers for $E_{c, h}$ on $V_{h}(\Omega)^{2}$. Thus, the constraint $v \in V_{h}(\Omega ;(-\infty, 1])$ is satisfied for the minimizers of $E_{c, h}$ over $V_{h}(\Omega)^{2}$.

A similar argument would show that $v(x) \geq 0$ a.e. in $\Omega$, and we conclude that all minimizers $(u, v)$ for $E_{c, h}$ over $V_{h}(\Omega)^{2}$ are such that $v(x) \in V_{h}(\Omega ;[0,1])$. Consequently, our choice of discrete functional renders the constraint on $v$ internal, which is not the case of the formulation proposed in [3]: if $(u, v)$ in a (constrained) minimizer for $E_{c, h}^{B}$ then $v(x) \geq 0$ a.e. and $E_{c, h}^{B}(u,-v)<E_{c, h}^{B}(u, v)$ as soon as $|\{x \in \Omega ; v(x)>0\}|>0$, so that the unconstrained minimization will never yield a non negative minimizer $v$ (if such a minimizer exists).

For a numerical standpoint, the absence of constraint on $v$ greatly simplifies the algorithm.

\subsection{Minimization strategy}

Consider the problem

$$
(u, v)=\min _{(u, v)}\left\{E_{c, h}(u, v) \mid(u, v) \in V_{h}(\Omega)^{2}\right\} .
$$

Because of the cross term $v^{2}|\nabla u|^{2}$, a direct finite element method cannot be used. Since $E_{c, h}$ is strictly convex in the directions $(\bullet, v)$ and $(u, \bullet)$, we propose an alternate minimization scheme; specifically, our algorithm is as follows: given $\left(u^{0}, v^{0}\right)$, compute $\left\{\begin{array}{l}u^{n}=\min _{u} E_{c, h}\left(u, v^{n-1}\right) \\ v^{n}=\min _{v} E_{c, h}\left(u^{n}, v\right)\end{array}\right.$ and set $E_{c}^{n}=E_{c}\left(u^{n}, v^{n}\right)$. 


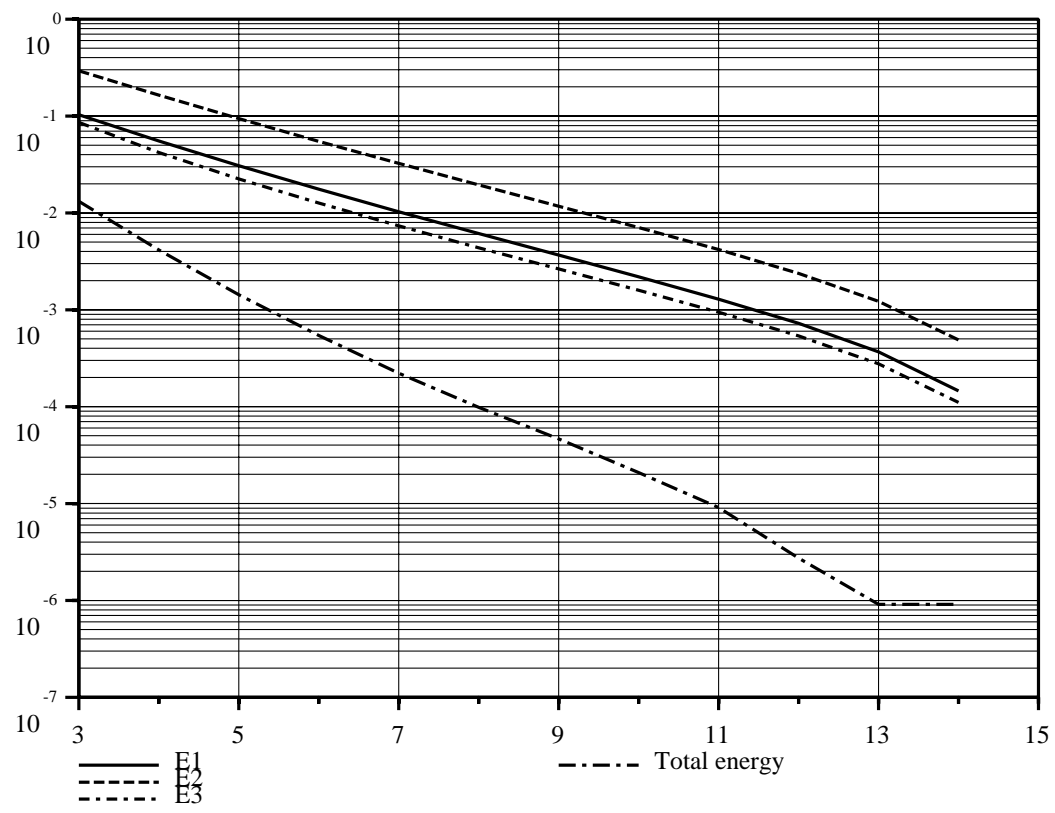

FiguRE 1. Relative error of the different parts of the functional.

TABlE 1. Computation on a $256 \times 256$ pixels images with $\alpha=5 \times 10^{-3}, \beta=5 \times 10^{-1}$, $c=10^{-1}$, without Jacobi preconditioning.

\begin{tabular}{|l|l|l|l|l|}
\hline Size of the subimages & Time & Alt. min. steps & C. G. steps $(u)$ & C. G. steps $(v)$ \\
\hline \hline $128 \times 128$ & $149 \mathrm{~s}$ & 9 & 225 & 233 \\
$64 \times 256$ & $82 \mathrm{~s}$ & 9 & 223 & 249 \\
\hline $64 \times 64$ & $85 \mathrm{~s}$ & 48 & 959 & 895 \\
$32 \times 128$ & $52 \mathrm{~s}$ & 48 & 1001 & 912 \\
$16 \times 256$ & $36 \mathrm{~s}$ & 48 & 1094 & 1003 \\
\hline $32 \times 32$ & $46 \mathrm{~s}$ & 218 & 3726 & 2867 \\
$16 \times 64$ & $31 \mathrm{~s}$ & 217 & 3793 & 2859 \\
\hline
\end{tabular}

TABLE 2. Computation on a $256 \times 256$ pixels images with $\alpha=5 \times 10^{-3}, \beta=5 \times 10^{-1}, c=10^{-1}$, with Jacobi preconditioning.

\begin{tabular}{|l|l|l|l|l|}
\hline Size of the subimages & Time & Alt. min. steps & C. G. steps $(u)$ & C. G. steps $(v)$ \\
\hline \hline $128 \times 128$ & $84 \mathrm{~s}$ & 9 & 121 & 73 \\
$64 \times 256$ & $49 \mathrm{~s}$ & 9 & 122 & 74 \\
\hline $64 \times 64$ & $57 \mathrm{~s}$ & 48 & 566 & 325 \\
$32 \times 128$ & $36 \mathrm{~s}$ & 48 & 559 & 319 \\
$16 \times 256$ & $26 \mathrm{~s}$ & 48 & 569 & 336 \\
\hline $32 \times 32$ & $36 \mathrm{~s}$ & 218 & 2237 & 1214 \\
$16 \times 64$ & $26 \mathrm{~s}$ & 217 & 2247 & 1216 \\
\hline
\end{tabular}




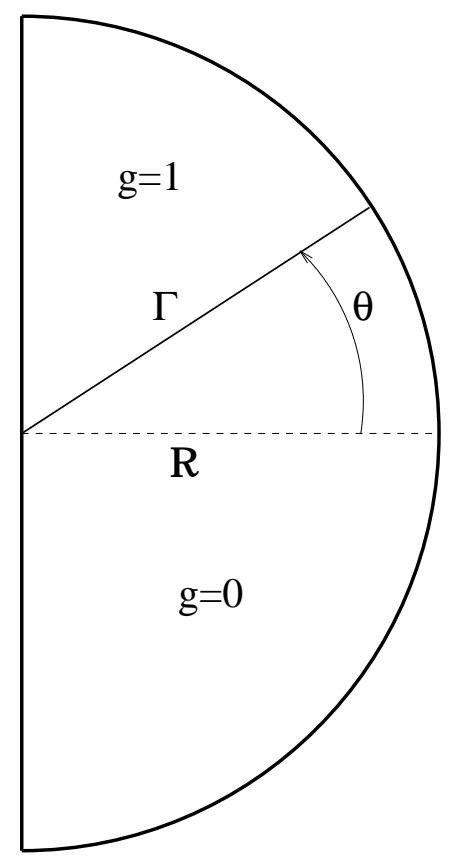

(a) Computational domain.

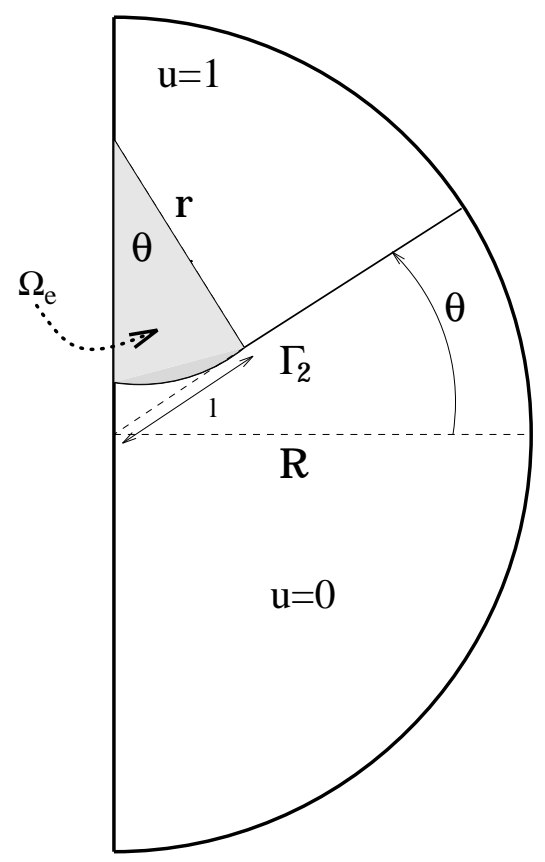

(b) Edge deformation.

FiguRE 2. Edge deformation across boundary.

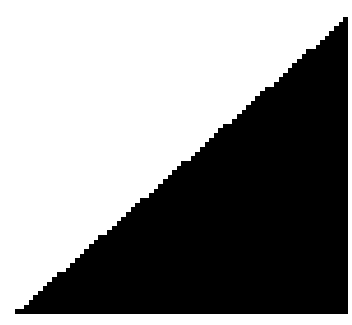

(a) Starting image.

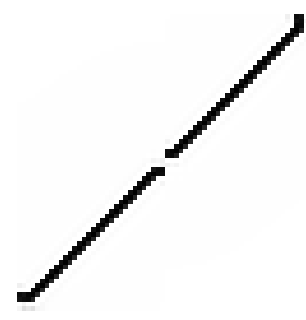

(b) Deformed edge set.

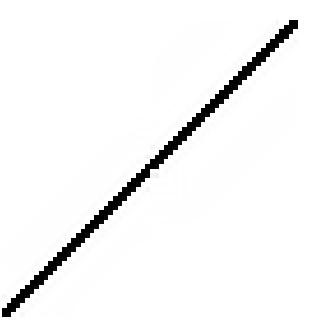

(c) Theoretical edge set.

FiguRE 3. Different behavior of edges across the subdomain boundary.

Since the successive minimization problems are strictly convex, the existence and uniqueness of $u_{n}$ and $v_{n}$ is ensured.

The successive minimization problems being strictly convex and quadratic, a straightforward finite element method can be implemented.

Remark. We have not managed, as of yet, to prove the convergence of the alternate minimization algorithm. Remark however that the sequence $E_{c}^{n}$ is decreasing.

In Figure 1, we present the evolution of the relative error for the different parts of $E_{c, h}$ for a computation on a real image of $256 \times 256$ pixels with the following parameters: $\alpha=2 \times 10^{-2}, \beta=5 \times 10^{-1}, c=10^{-1}$. 

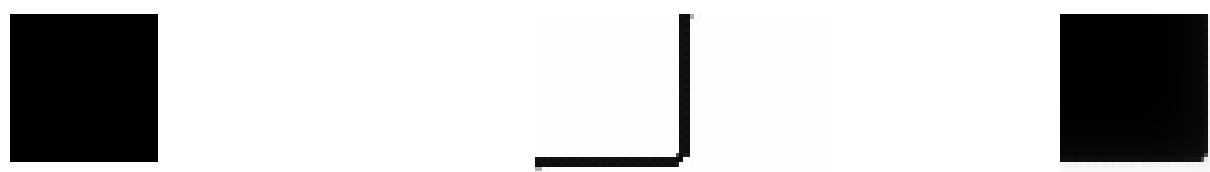
(a) Starting image.
(b) Edge set " $v "$.
(c) Soft image " $u$ "

Figure 4. Angle with $c=4 \times 10^{-2}, \alpha=5 \times 10^{-3}, \beta=2 \times 10^{-2}, k_{c}=5 \times 10^{-3}$.

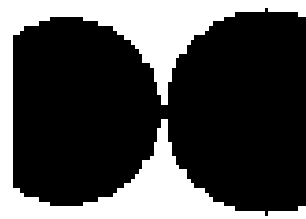

(a) Starting image.

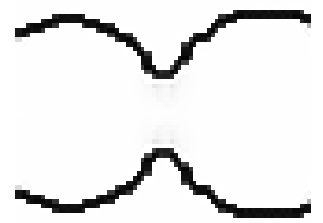

(b) Edge set " $v "$.

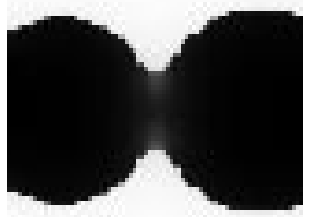

(c) Soft image " $u$ ".

FiguRE 5. Cuspidal point with $c=4 \times 10^{-2}, \alpha=5 \times 10^{-3}, \beta=2 \times 10^{-2}, k_{c}=5 \times 10^{-3}$.
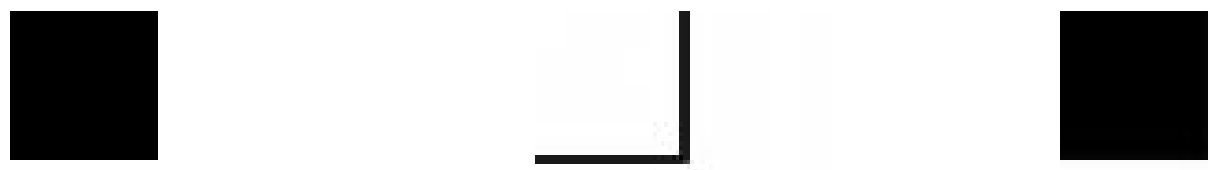
(a) Starting image.
(b) Edge set " $v$ ".
(c) Soft image " $u$ ".

FiguRE 6. Square angle with $c=4 \times 10^{-3}, \alpha=2 \times 10^{-1}, \beta=5 \times 10^{-1}$.

The data plotted is $\frac{\left|E_{i}(t)-E_{i}(\infty)\right|}{E_{i}(\infty)}$ where $E_{i}(t)$ is the part $E_{i}$ of $E_{c, h}$, according to the following notations, at the step $t$ of the alternates directions scheme. The labels are: $E_{1}=\int_{\Omega}\left(v^{2}+k_{c}\right)|\nabla u|^{2} d x, E_{2}=$ $\alpha \int_{\Omega}\left(c|\nabla v|^{2}+\frac{(1-v)^{2}}{4 c}\right) d x$, and $E_{3}=\beta \int_{\Omega}\left|u-g_{c, h}\right|^{2} d x$.

\subsection{Parameter choice}

We now describe the choice of the discretization parameter $h$, according to the hypothesis $h=o(c)$, as well as that of the pixel coordinates. 


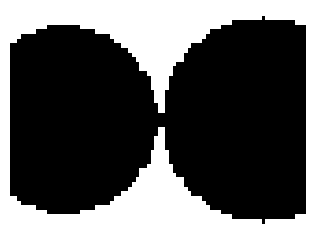

(a) Starting image.

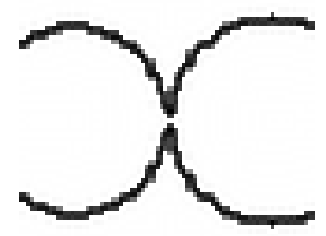

(b) Edge set " $v$ ".

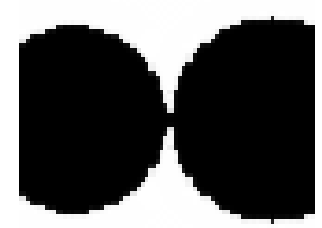

(c) Soft image " $u$ ".

Figure 7. Cuspidal point with $c=4 \times 10^{-3}, \alpha=2 \times 10^{-2}, \beta=1$.

Let $\Omega$, be the original domain, and set $\hat{x}=(1 / k) \cdot x \in \hat{\Omega}=k \cdot \Omega$, for all $x \in \Omega$, then one has

$$
\begin{aligned}
E_{c}(u(x), v(x))=\int_{\hat{\Omega}}\left(\hat{v}^{2}+k_{c}\right)|\nabla \hat{u}|^{2} d \hat{x} & +\alpha \int_{\hat{\Omega}}\left(c|\hat{\nabla} v|^{2}+\frac{(1-\hat{v})^{2}}{4 . k^{2} \cdot c}\right) d \hat{x} \\
& +\frac{\beta}{k^{2}} \int_{\hat{\Omega}}(\hat{u}-\hat{g})^{2} d \hat{x} \\
=\int_{\hat{\Omega}}\left(\hat{v}^{2}+k_{c}\right)|\nabla \hat{u}|^{2} d \hat{x} \quad & +\frac{\alpha}{k} \int_{\hat{\Omega}}\left(c . k|\hat{\nabla} v|^{2}+\frac{(1-\hat{v})^{2}}{4 . k . c}\right) d \hat{x} \\
& +\frac{\beta}{k^{2}} \int_{\hat{\Omega}}(\hat{u}-\hat{g})^{2} d \hat{x} .
\end{aligned}
$$

The magnitude ratio between the different terms in $E_{c}$ will only be preserved for all homothetics of ratio $k$ of the original domain if

$$
\left\{\begin{array}{l}
c(k)=c . k \\
\alpha(k)=\frac{\alpha}{k} \\
\beta(k)=\frac{\beta}{k^{2}} .
\end{array}\right.
$$

Thus the domain size $k$ influences both the choice of allowable discretization parameter $h$ and of the constants $\alpha$ and $\beta$. In other words, differents computation cannot be compared until $\alpha, \beta$ and $k$ are specified.

\subsection{General choices}

No computation can be performed without prior mesh generation. A simplifying idea would be to use regular meshes (grids), the nodes of which coincide with the pixels. The finite element method could then be equivalent to a finite difference method, provided all elements are identical and identically oriented. This lowers the computation time, since approximate integration or affine transformation are then superfluous. In a finite element method, however, the computation time is mostly spent solving the linear system, while the time spent generating more complicated finite element matrices is negligible; this motivates our decision to generate arbitrary meshes that do not need to coincide with the pixels.

With arbitrary mesh, the implementation of further enhancements like scale focusing, mesh adaptation, and the processing of non rectangular images becomes possible. For the same reason, it would be possible to use higher order elements, if one could prove the convergence of the discrete functional for a better discretization.

In the presented computations, however, the computational mesh has been taken to coïncide with the pixels (but the possible simplification resulting from the use of such a mesh have not been implemented; in other words, we are still using a general finite element algorithm for the solving of the minimization problem). In any case, $h$, defined as the radius of the inscribed circle in an element is thus of the order of 1 , while $k$ (the size of the image) is proportional to the square root of the number of pixels. 


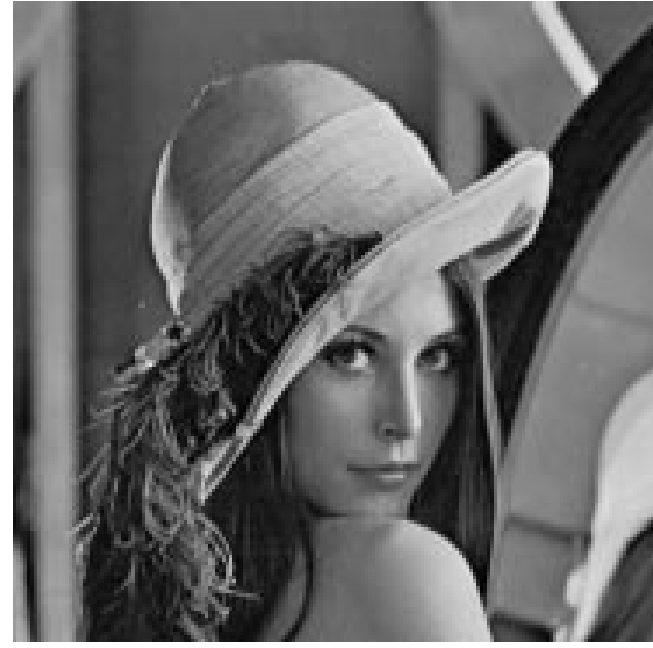

(a) Starting image, $480 \times 480$ pixels.

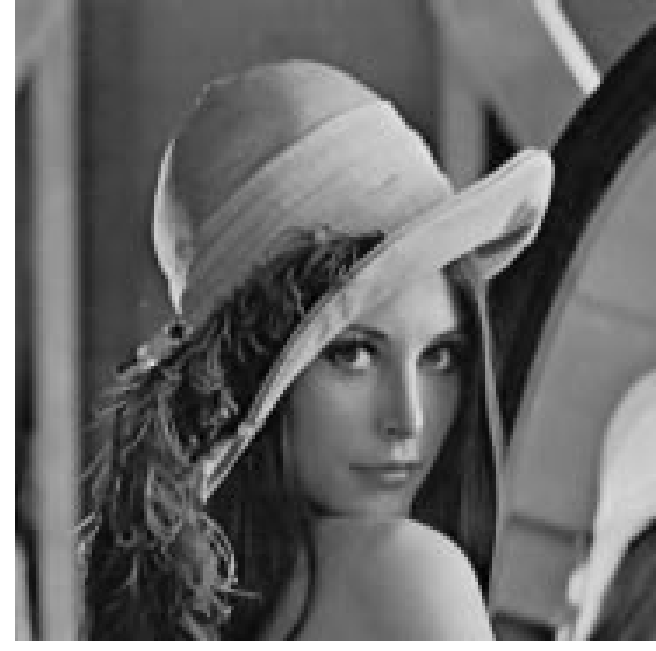

(b) Soft image " $u$ ".

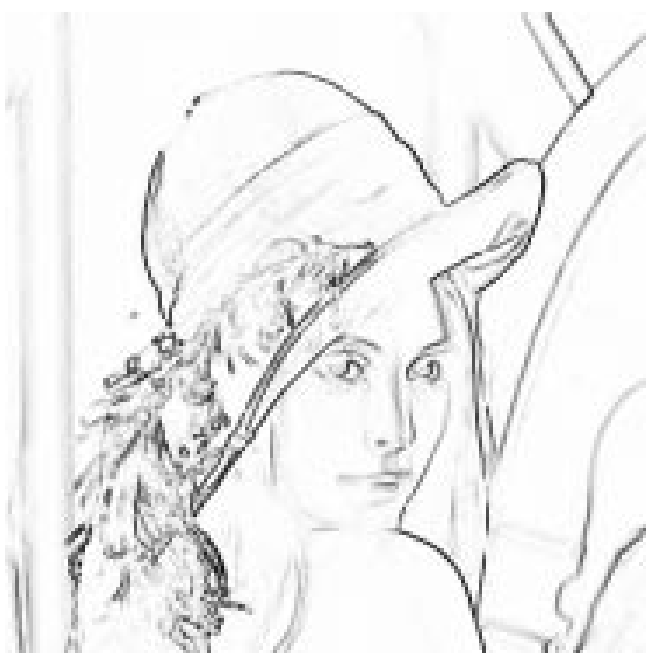

(c) Edge set " $v "$.

Figure 8. Computation for $\alpha=5 \times 10^{-3}, \beta=5 \times 10^{-1}, c=10^{-1}$.

Each alternate minimization step involves the resolution of a linear system, the dimension of which is the number of nodes.

Since finite element method, produces linear system whose matrices are symmetrical with a large proportion of zeros, a skyline storage is implemented: the only terms to be stored as a vector are the elements of each line between the first non logically zero term and the diagonal term. A second vector, the profile, keeps the position in the compressed vector of the diagonal terms of the starting matrix.

Computations are then performed only inside the skyline, which enhances the computational speed too. 


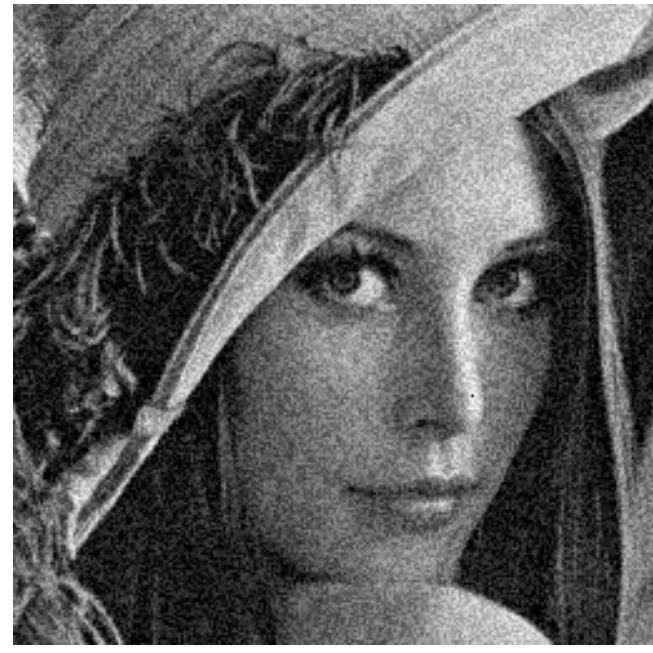

(a) Starting image, $256 \times 256$ pixels.

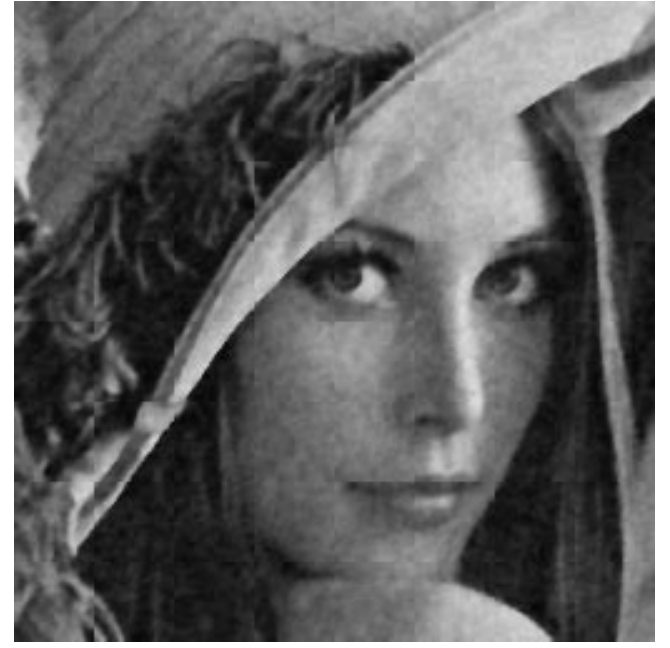

(b) Soft image " $u$ ".

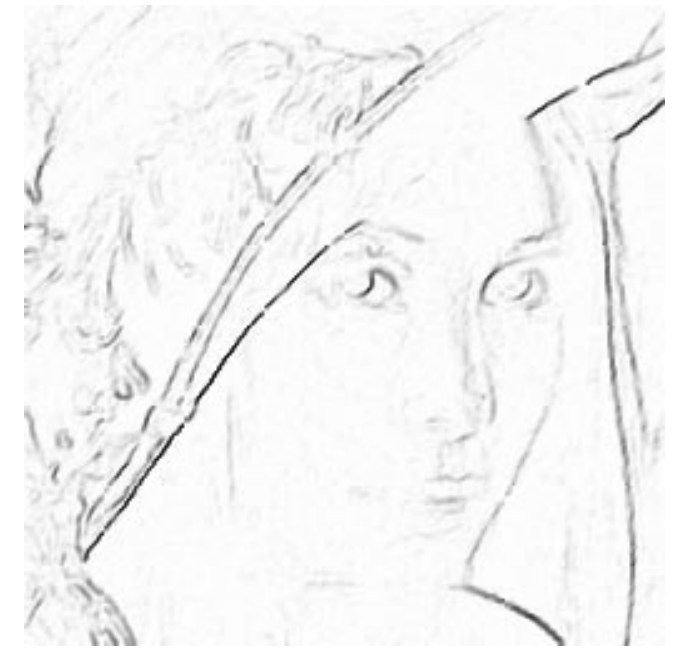

(c) Edge set " $v "$.

Figure 9. Computation for $\alpha=2 \times 10^{-2}, \beta=5 \times 10^{-1}, c=10^{-1}$.

The linear system resolution algorithm we use is a conjugated gradient method. For this algorithm, a starting point is required, and we use the results of the former alternate minimization step, considering this way that the convergence rate of the alternate minimization is low enough to produce results close one from another.

A second enhancement is the implementation of a Jacobi (diagonal) preconditioning, inside the conjugate gradient algorithm: the conjugate gradient direction for the resolution of $A . x=b$ is that of $D^{-1} \cdot A \cdot D^{-t}$, instead of that of $A$, where $[D]_{i, j}=\delta_{i, j} \sqrt{\left[A_{i, j}\right]}$. The Jacobi preconditioning reduces the number of conjugate steps by a factor close to 2 for the $u$-problem and 3 for the $v$-problem. 


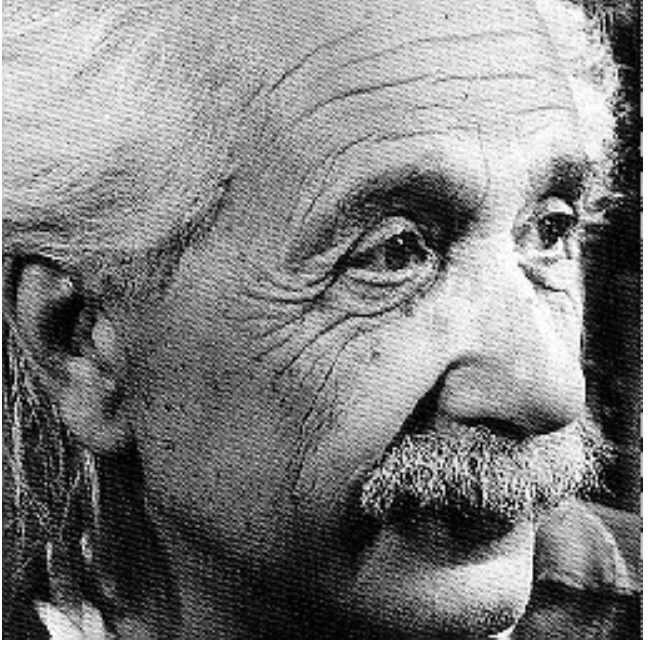

(a) Starting image, $256 \times 256$ pixels.

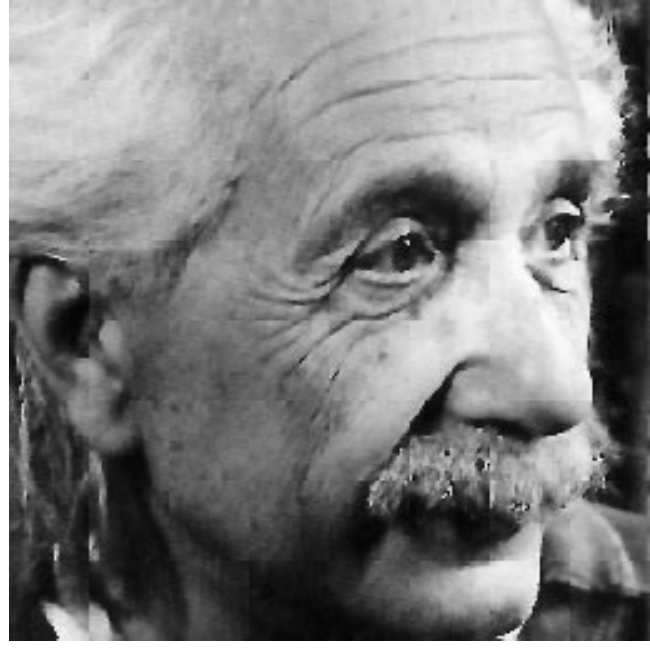

(b) Soft image " $u$ ".

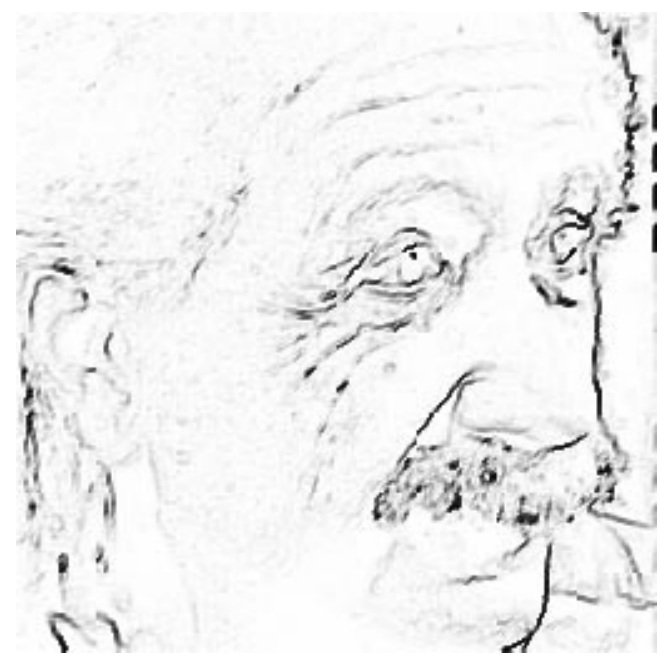

(c) Edge set " $v$ ".

Figure 10. Computation for $\alpha=2 \times 10^{-2}, \beta=5 \times 10^{-1}, c=10^{-1}$.

At last, we need a stopping criterion for the alternate minimization. Since we didn't manage to prove the convergence of this scheme, we can't use an error majoration, then we decided to use the distance between the results of successive alternate minimization results, i.e. the norm of $v_{n}-v_{n-1}$ in $H^{1}, L^{2}$ or $L^{\infty}$, as desired, with an upper bound for the total number of alternate minimization steps. 


\subsection{Domain decomposition}

Since both computational time and memory requirement dependence on the number of pixels are more than linear, for finite element method, we implemented a domain decomposition method: each image is decomposed into sub-images and the minimization of $E_{c, h}$ is computed on each subimage.

The difficulty in such an algorithm resides in the lack of continuity of $u$ and $v$ on subdomain boundaries: deformations could then appear across the subdomain boundaries. We show in the next section how to control the edges deformation across subdomain boundaries, but the lack of continuity of $u$ should, in truth, necessitate a better decomposition algorithm, which may use, for example, overlapping subdomains.

In Table 1 and 2, we present some computational time and parameter for a 256 by 256 image, with several decomposition.

Table 1 is related on computations without preconditioning, and Table 2 is related on computations with a Jacobi preconditioning.

It is important to remark that even with those enhancements, between 50 and $75 \%$ of the computation time is spent in implementing the conjugate gradient method: thus any improvement in the linear system resolution results in an important decrease in computational time.

\section{Numerical RESUlts}

\subsection{Edge geometry}

The following regularity conjectures on the minimizing set $\Gamma$ for $\mathcal{E}$ can be found in [12] Theorem 2.1, p. 599:

- $\Gamma$ meets $\partial \Omega$ perpendicularly,

- a point $P \in \Omega$, is the end of 0,1 or 3 edges (i.e. corners, cuspidal points and cross point are not allowed), and if $P$ is a triple point, the arcs meet with angles $2 \pi / 3$.

Those geometrical restrictions lead to two kinds of edge deformations: across the subdomain boundaries and at edge corners or intersection inside each subdomain. In view to decompose the domain into several subdomains, we want to prevent from the first effect.

We now focus to the intersection of a sub domain and a circle of radius $R$, centered at the point where an edge, $\Gamma$, crosses the boundary. We suppose that $\Gamma$ is a segment, crossing the boundary into an angle $\theta$ and that $g$ equals 1 , above $\Gamma$ and 0 , otherwise (see Fig. 2 a for the geometrical construction). Then, we build a second edge set, denoted by $\Gamma_{2}$, derived from $\Gamma$ and such that from a distance $l$ from the boundary, $\Gamma$ has been replaced by an arc of radius $r$, crossing the boundary perpendicularly (see Fig. $2 \mathrm{~b}$ for the geometrical construction). By imposing that the arc is tangent to the theoretical edge, we get $r=l \cdot \cot \theta$.

Let $\mathcal{E}_{1}$ and $\mathcal{E}_{2}$ be the total energy of each configuration; then $\mathcal{E}_{1}=R . \alpha$, while since $\left|\Gamma_{2}\right|=R-l .(1-\theta \cdot \cot \theta)$ and $\left|\Omega_{e}\right|=\frac{r . l}{2}-\frac{r^{2}}{2} \theta, \mathcal{E}_{2}=\frac{l^{2} \cdot \beta \cdot \cot \theta}{2} .(1-\theta \cdot \cot \theta)-l . \alpha .(1-\theta \cdot \cot \theta)+R . \alpha$.

We now minimize $\mathcal{E}_{2}$ over all $l \leq R$ : if $R \geq \frac{\alpha}{\beta} \tan \theta$, the minimum value is reached for $l=\frac{\alpha}{\beta} \tan \theta$ and is equal to $R . \alpha-\frac{\alpha^{2}}{\beta}(\tan \theta-\theta)$. Since, for $\theta \in\left[0, \frac{\pi}{2}\right], \tan \theta-\theta \geq 0$, we obtain $\mathcal{E}_{2} \leq \mathcal{E}_{1}$, and the detected edge set will be different from the theoretical one at the point where it crosses the subdomain boundaries, if not perpendicular.

In Figure 3a, we decomposed the image of Figure 3b into two vertical bands; the resulting deformation of the edge is demonstrated.

One can however estimate the radius of the area where such deformation appears as a linear function of $\alpha / \beta$ (see the expression of the optimal $l$ above). Then, a proper choice of $\alpha / \beta$ should be such that the radius is less than the width of a pixel; in such a case, the edge will take its theoretical shape (see Figure $3 \mathrm{c}$ ).

The resolution parameters in Figure 3 are for (b): $\alpha=5 \times 10^{-3}, \beta=2 \times 10^{-2}$ and $c=4 \times 10^{-2}$, and for (c): $\alpha=10^{-3}, \beta=5 \times 10^{-1}$ and $c=4 \times 10^{-2}$.

A similar argument can be used to show that there are no corner or cuspidal point allowed for the optimal edge set but that, with a proper adjustment of the parameters $\alpha$ and $\beta$ the deformation can be controlled and inscribed inside a pixel. 
Figures 4 and 5 show deformed corners and cuspidal points for the set of parameters $\alpha=5 \times 10^{-3}, \beta=2 \times 10^{-2}$ and $c=4 \times 10^{-2}$, while Figures 6 and 7 show results based on the same images, with adjusted parameters, so as to generate the real edge geometry.

In Figure 8, we present a numerical result for a standard image, "Lenna" (a), the soft image, corresponding to $u$ (b) and the edge set $v(\mathrm{c})$.

In Figure 9a, we magnify a detail from Figure 8a and perturb it with random noise (salt and pepper noise with maximal intensity \pm 80 ); the results are presented in Figures $9 \mathrm{~b}$ and c; they demonstrate the sturdiness of the algorithm when subject to random noise.

In Figure 10a, we took a rastered portrait of A. Einstein (magnification of a printed image) and used our algorithm. The results are shown in Figure 10b and 10c; they are unaffected by periodical perturbations.

The author would like to thank A. Chambolle and G. Francfort for their helpful advices.

\section{REFERENCES}

[1] L. Ambrosio and V.M. Tortorelli, Approximation of functionals depending on jumps by elliptic functionals via $\Gamma$-convergence. Comm. Pure Appl. Math. 43 (1990) 999-1036.

[2] L. Ambrosio and V.M. Tortorelli, On the approximation of free discontinuity problems. Boll. Un. Mat. Ital. VI-B (1992) $105-123$.

[3] G. Belletini and A. Coscia, Discrete approximation of a free discontinuity problem. Num. Funct. Anal. Optim. 15 (1994) $201-224$.

[4] A. Bonnet, On the regularity of the edge set of Mumford-Shah minimizers. Prog. in Nonlinear Differential Equation and Their Applications 25 (1996) 93-103.

[5] H. Brezis, Analyse fonctionnelle. Masson (1989).

[6] A. Chambolle, Image segmentation by variational methods: Mumford and Shah functional and the discrete approximations. SIAM J. Appl. Math. 55 (1995) 827-863.

[7] P.G. Ciarlet, The finite element method for elliptic problems. North-Holland (1987).

[8] E. De-Giorgi, M. Carriero and A. Leaci, Existence theorem for a minimum problem with free discontinuity set. Arch. Rational Mech. Anal. 108 (1989) 195-218.

[9] F. Dibos and E. Séré, An approximation result for the minimizers of the Mumford-Shah functional. Boll. Un. Mat. Ital. A 11 (1997).

[10] L.C. Evans and R. Gariepy, Measure theory and fine properties of functions. CRC Press, Boca Raton (1992).

[11] S. Finzi-Vita and P. Perugia, Some numerical experiments on the variational approach to image segmentation, in Proc. of the Second European Workshop on Image Processing and Mean Curvature Motion, Palma de Mallorca (1995) 233-240.

[12] D. Mumford and J. Shah, Optimal approximation by piecewise smooth functions and associated variational problems. Comm. Pure Appl. Math. XLII (1989) 577-685.

[13] T.J. Richardson and S.K. Mitter, A variational formulation based edge focusing algorithm. Sadhana Acad. P. Eng. S. 22 (1997) 553-574. 